\title{
Flow-induced anisotropic viscosity in short FRPs
}

\author{
Róbert Bertóti and Thomas Böhlke*
}

\begin{abstract}
Background: The commonly used flow models for Fiber Reinforced Polymers (FRPs) often neglect the flow-induced anisotropy of the suspension, but with increasing fiber volume fraction, this plays an important role. There exist already some models which count on this effect. They are, however, phenomenological and need a fitted model parameter. In this paper, a micromechanically-based constitutive law is proposed which considers the flow-induced anisotropic viscosity of the fiber suspension.

Methods: The introduced viscosity tensor can handle arbitrary anisotropy of the fluid-fiber suspension which depends on the actual fiber orientation distribution. Assuming incompressible material behaviour, a homogenization method for unidirectional structures in contribution with orientation averaging is used to determine the effective viscosity tensor. The motion of rigid ellipsoidal fibers induced by the flow of the matrix material is described based on Jeffery's equation. The reorientation of the fibers is modeled in two ways: by describing them with fiber orientation vectors, and by fiber orientation tensors. A numerical implementation of the introduced model is applied to representative flow modes.

Results: The predicted effective stress values depending on the actual fiber orientation distribution through the anisotropic viscosity are analyzed in transient and stationary flow cases. In the case of the assumed incompressibility, they show similar effective viscous material behaviour as the results obtained by the use of the Dinh-Armstrong constitutive law.
\end{abstract}

Conclusions: The introduced model is a possible way to describe the flow-induced anisotropic viscosity of a fluid-fiber suspension based on the mean field theory.

Keywords: Anisotropic viscosity, Homogenization, Jeffery's equation

\section{Background}

The orientation distribution of discontinuous fibers in a composite has a considerable impact on the mechanical properties of the reinforced material. To forecast the final fiber orientation distribution of a produced part, the flow process has to be simulated. Polymer-composites containing short fiber reinforcements are often produced through injection molding. Nowadays, with the compression molding technology also discontinuous long fiber reinforced polymer parts can be produced.

The motivation of this work is to set up a micromechanically-based rheological model which can describe the flow-induced effective anisotropic viscous

*Correspondence: thomas.boehlke@kit.edu

Chair for Continuum Mechanics, Institute of Engineering Mechanics, Karlsruhe Institute of Technology (KIT), Kaiserstraße 10, 76131 Karlsruhe, Germany behaviour of a fiber suspension, depending on the fiber orientation distribution in the fluid.

To describe the orientation state and the dynamics of short rigid fibers in a numerically efficient way, Advani and Tucker (1987) introduced the fiber orientation tensors of the first kind (Kanatani 1984). Based on Jeffery's Equation (Jeffery 1922; Junk and Illner 2007), the motion of a single rigid fiber in a Newtonian fluid and the change of the orientation tensors can be described. In the literature, for many fiber systems, the fiber-fiber interactions are modeled in different ways. The most often used models are the Folgar-Tucker (1984), the Reduced Strain Closure (Wang et al. 2008) and the Anisotropic Rotary Diffusion (Phelps and Tucker III 2009). All of them are already implemented in commercial software packages.

The rheology of fiber suspensions is also described by various models in the literature. If the molten composite 
is considered to be linear and isotropic, then the viscosity model used for Newtonian fluids is applicable. It would be then sufficient to determine the effective scalar viscosity of the mixture which can be measured, e.g., with a shear rheometer.

But polymer melts show a strongly nonlinear behaviour, see, e.g., (Ferry 1980). The stress is not directly proportional to the strain rate, even not for pure molten polymers. Considering filled polymer melts, the nonlinear stress-strain rate relation is described, e.g., by (Poslinski et al. 1988). In commercial software, for modeling the flow of fiber reinforced polymer melts, the shear thinning behaviour of the fiber-fluid mixture is implemented, but the anisotropy of the suspension is mostly neglected.

There are also constitutive laws which consider the anisotropic flow properties of fiber suspensions. One of them is the Transversely Isotropic Fluid (TIF) model from Ericksen (1960). The Phan-Thien-Graham model (Phan-Thien and Graham 1991) is a modification of the TIF model and it has the same structure as the DinhArmstrong model (Dinh and Armstrong 1984). Both of them depend linearly on the fourth order fiber orientation tensor.

The objective of this paper is to propose a flowinduced anisotropic viscosity model which depends on the complete orientation information of the fibers. The introduced method is based on a homogenization scheme (Willis 1977), combined with orientation averaging (Advani and Tucker III 1987). The fully coupled fluid-fiber interaction is described. The evolution of the fiber orientation distribution, caused by the flow, is investigated. The number of model parameters is kept minimal in order to reduce (future) measurement costs, fitting efforts and uncertainties. A three-dimensional numerical implementation of the presented model can be done similar to (Ospald 2014) or (Schneider et al. 2015). The foregoing aspects contribute to the development of the mold filling simulations of fiber reinforced materials.

\section{Methods}

\section{Model equations}

\section{Effective stress in the case of incompressibility}

It is assumed that the fluid-fiber suspension is incompressible. The effective, i.e. macroscopic, Cauchy stress $\overline{\boldsymbol{\sigma}}$ is additively decomposed into a pressure-dependent spherical and a pressure-independent deviatoric part

$$
\overline{\boldsymbol{\sigma}}=-\bar{p} \boldsymbol{I}+\overline{\boldsymbol{\sigma}}^{\prime}
$$

In this paper, only the pressure-independent deviatoric part of the Cauchy stress $\overline{\boldsymbol{\sigma}}^{\prime}$ is investigated, since $\bar{p}$ is a reaction force and follows from the boundary conditions.

\section{Effective viscosity tensor}

It is assumed that the effective viscous material behaviour is physically linear. In such a case, the deviatoric effective Cauchy stress $\overline{\boldsymbol{\sigma}}^{\prime}$ is given by a linear mapping of the deviatoric effective strain rate $\overline{\boldsymbol{D}}^{\prime}$ being the symmetric part of the deviatoric effective velocity gradient

$$
\overline{\boldsymbol{\sigma}}^{\prime}=\overline{\mathbb{V}}\left[\overline{\boldsymbol{D}}^{\prime}\right] .
$$

The assumption of a linear viscosity is a severe restriction, because the molten polymer matrix and so the matrix-fiber suspension show a strongly non-Newtonian behaviour. Nonetheless, it is introduced due to the fact that the homogenization of nonlinear material properties is much more difficult. Hence, as a first step a physically linear model is considered. The effective viscosity tensor $\overline{\mathbb{V}}$ is of fourth order which can take into account an arbitrary anisotropy of the fluid-fiber suspension. $\overline{\mathbb{V}}$ depends on both the matrix behaviour, assumed to be linear viscous, and on the geometry, orientation distribution, and arrangement of the fibers. In the following, the effective viscosity tensor is estimated using a mean field theory taking into account the linear viscous behaviour of the matrix material, the geometry of the fibers, and their orientation distribution. The interaction of fibers is not explicitly considered. For the notation of the fourth order viscosity tensor which has both the minor and the major symmetry, the normalized Voigt notation is used (see, e.g., (Cowin and Mehrabadi 1987)) with which the tensor can be represented by a symmetric six times six matrix

$$
\overline{\mathbb{V}} \hat{=}\left(\begin{array}{cccccc}
\bar{V}_{11} & \bar{V}_{12} & \bar{V}_{13} & \bar{V}_{14} & \bar{V}_{15} & \bar{V}_{16} \\
& \bar{V}_{22} & \bar{V}_{23} & \bar{V}_{24} & \bar{V}_{25} & \bar{V}_{26} \\
& & \bar{V}_{33} & \bar{V}_{34} & \bar{V}_{35} & \bar{V}_{36} \\
& & & \bar{V}_{44} & \bar{V}_{45} & \bar{V}_{46} \\
& & & & \bar{V}_{55} & \bar{V}_{56} \\
& \text { sym. } & & & & \bar{V}_{66}
\end{array}\right) .
$$

\section{Homogenization for the effective viscosity tensor}

For estimating the effective linear viscosity tensor by means of a mean field theory, the microstructure of the fluid-fiber suspension is imaginarily decomposed into unidirectional pseudo-domains similarly as in (Kammoun et al. 2011). The pseudo-domains model one fiber and the matrix environment. To each fiber $\alpha$ belongs such a unidirectional pseudo-domain, with a transversely isotropic (uni-directional) viscosity tensor $\overline{\mathbb{V} \sim} \sim_{\alpha}^{\text {ud }}$ which belongs to the family of TIF models. The overall effective linear viscosity tensor $\overline{\mathbb{V}}$ is obtained by the orientation average of the unidirectional viscosity tensors. The discrete version of orientation averaging with equal weighted representative orientations is used, i.e. it is assumed that the fibers have a constant aspect ratio and a constant volume fraction. The number of fibers is denoted with $M$. In this case 


$$
\overline{\mathbb{V}}=\frac{1}{M} \sum_{\alpha=1}^{M} \overline{\mathbb{V}}_{\alpha}^{\mathrm{ud}}
$$

The short fibers are considered to be rigid, which is ensured in the model by infinite fiber viscosities.

Based on this assumption, each $\overline{\mathbb{V}}_{\alpha}^{\text {ud }}$ is approximated with the Hashin-Shtrikman lower bound (Willis 1977)

$$
\overline{\mathbb{V}}_{\alpha}^{\mathrm{ud}}=\mathbb{V}^{\mathrm{m}}+\frac{c_{\mathrm{f}}}{c_{\mathrm{m}}} \mathbb{P}_{\alpha}^{-1}
$$

where $c_{\mathrm{f}}$ is the fiber- and $c_{\mathrm{m}}$ is the matrix volume fraction. $\mathbb{P}_{\alpha}$ is the polarization tensor described, e.g., by Walpole (1969) and Ponte Castaneda (1998) depending on the matrix material properties and on the geometry and the orientation of the fiber $\alpha$. The properties of the matrix viscosity $\mathbb{V}^{\mathrm{m}}$ are discussed later.

The advantage of the Hashin-Shtrikman homogenization method is that it is derived based on the variational principle (Willis 1977), and the lower bound gives a finite effective viscosity for the unidirectional pseudo-domain, also in the case of infinitely viscous, i.e. rigid, fibers. The disadvantage of this method is that we get only a lower bound. The orientation averaging (4) gives a Voigt-type average which is an upper bound for the dissipation.

\section{Matrix behaviour}

The viscous behaviour of the matrix is assumed to be linear, isotropic and incompressible given by $\sigma^{\mathrm{m}^{\prime}}=$ $\mathbb{V}^{\mathrm{m}}\left[\boldsymbol{D}^{\mathrm{m}^{\prime}}\right]$ with the isotropic matrix viscosity

$$
\mathbb{V}^{\mathrm{m}}=3 \eta_{\mathrm{v}} \mathbb{P}_{1}+2 \eta_{\mathrm{s}} \mathbb{P}_{2},
$$

where $\eta_{\mathrm{v}}$ is the volume- and $\eta_{\mathrm{s}}$ is the shear viscosity of the matrix material. The tensor $\mathbb{P}_{1}=I \otimes I / 3$ and $\mathbb{P}_{2}=$ $\mathbb{I}^{\mathrm{s}}-\mathbb{P}_{1}$ are the first- and the second projector tensors of fourth order with major and minor symmetry, $\boldsymbol{I}$ is the second order unit tensor, and $\mathbb{I}^{\mathrm{s}}$ is the symmetric part of the fourth order unit tensor. Because of the assumed incompressibility, only the $\mathbb{P}_{2}$ term of the matrix viscosity tensor is relevant, the $\mathbb{P}_{1}$ term cancels out through $\overline{\boldsymbol{D}}^{\prime}$. This induces that the volume viscosity $\eta_{\mathrm{v}}$ in $\mathbb{V}^{\mathrm{m}}$ has no influence in the case of incompressibility. From now on, the notation $\mathbb{V}^{\mathrm{m} \prime}=\eta_{\mathrm{s}} \mathbb{P}_{2}$ is used. The polarization tensor $\mathbb{P}_{\alpha}$ depends in general also on $\eta_{\mathrm{v}}$. For the case of incompressibility, the effect of $\eta_{\mathrm{v}}$ can not be filtrated from $\mathbb{P}_{\alpha}$ as simply as from $\mathbb{V}^{\mathrm{m}}$. To ensure the incompressibility, the limit of $\mathbb{P}_{\alpha}$ is calculated for the case that $\eta_{\mathrm{v}}$ goes to infinity. This limit results a singular polarization tensor $\mathbb{P}_{\alpha}^{\prime}$ which is inverted on the non-singular subspace on traceless tensors for (5). The consequent notation of $\overline{\mathbb{V}}$ for the incompressible case would be $\overline{\mathbb{V}}^{\prime}$, but the prime is dropped for simpler notation.

\section{Fiber orientation and reorientation}

A short rigid fiber is described by a normalized orientation vector $\boldsymbol{n}=n_{i} \boldsymbol{e}_{i}$, where $n_{i}$ are the vector components and $\boldsymbol{e}_{i}$ the ortho-normal basis vectors. The orientationchange of an ellipsoidal rigid fiber $\boldsymbol{n}_{\alpha}$ immersed in a Newtonian-flow, is described by Jeffery's Equation (Jeffery 1922; Junk and Illner 2007)

$$
\begin{gathered}
\dot{\boldsymbol{n}}_{\alpha}=\overline{\boldsymbol{W}}\left[\boldsymbol{n}_{\alpha}\right]+\bar{\xi}\left(\overline{\boldsymbol{D}}\left[\boldsymbol{n}_{\alpha}\right]-\left(\boldsymbol{n}_{\alpha} \otimes \boldsymbol{n}_{\alpha} \otimes \boldsymbol{n}_{\alpha}\right)[\overline{\boldsymbol{D}}]\right), \\
\bar{\xi}=\frac{\bar{a}^{2}-1}{\bar{a}^{2}+1} \quad, \quad \bar{a}=\bar{l} / \bar{d},
\end{gathered}
$$

where $\alpha=1,2, \ldots, M$ is the index of the fibers. $\bar{W}$ is the effective vorticity tensor, and $\bar{D}$ is the effective strain rate tensor, being the antisymmetric and the symmetric parts of the effective velocity gradient $\overline{\boldsymbol{L}}=\partial \overline{\boldsymbol{v}} / \partial \boldsymbol{x}$, respectively. $\bar{\xi}$ is a geometry parameter of a representative fiber, determined through the aspect-ratio $\bar{a}$, the length $\bar{l}$, and the diameter $\bar{d}$. With the use of the dilute distribution approximation, which assumes that the fibers do not have any interaction with each other, Jeffery's equation separately describes the motion of all $M$ pieces of fibers.

A statistical prediction of the fiber orientation state is of interest. The statistical description of the fiber orientation state is exactly given by the fiber orientation distribution function (FODF) (Advani and Tucker III 1987; Kanatani 1984). Numerically, it is more efficient to approximate the FODF with fiber orientation tensors. Advani and Tucker (1987) defined the reducible, second- and fourth order fiber orientation tensors of the first kind (Kanatani 1984) as

$$
\overline{\boldsymbol{N}}=\frac{1}{M} \sum_{\alpha=1}^{M} \boldsymbol{n}_{\alpha} \otimes \boldsymbol{n}_{\alpha} \quad, \quad \overline{\mathbb{N}}=\frac{1}{M} \sum_{\alpha=1}^{M} \boldsymbol{n}_{\alpha} \otimes \boldsymbol{n}_{\alpha} \otimes \boldsymbol{n}_{\alpha} \otimes \boldsymbol{n}_{\alpha} .
$$

These orientation tensors are not only used to describe the actual fiber orientation state (in terms of the leading moments of the FODF), but through the extension of Jeffery's Eq. (7), the change of the fiber orientation state is directly approximated by the orientation tensors

$$
\dot{\bar{N}}=\bar{W} \bar{N}-\bar{N} \bar{W}+\bar{\xi}(\bar{D} \bar{N}+\bar{N} \bar{D}-2 \overline{\mathbb{N}}[\bar{D}]) .
$$

In the evolution equation of the second order fiber orientation tensor $\bar{N}$, the fourth order fiber orientation tensor $\overline{\mathbb{N}}$ appears. Analogously, in the evolution equation of $\overline{\mathbb{N}}$, the next higher even order fiber orientation tensor appears. Instead of the evolution equation of $\overline{\mathbb{N}}$, the invariant-based optimal fitting (IBOF) closure (Chung et al. 2002) is used to approximate the values of $\overline{\mathbb{N}}$ in each time step, based on the invariants of $\bar{N}$.

With the use of the fiber orientation tensors, the orientation evolution (7) can be calculated more efficiently (9). Similarly, the orientation averaging (4) can also be done numerically in a more efficient way with the help of the 
fiber orientation tensors, as it is described in (Advani and Tucker III 1987).

The evolution of $\bar{N}$ (9) can be calculated also with the Fast Exact Closure (FEC) (Montgomery-Smith et al. 2011). With the FEC, the exact solution of Jeffery's equation is obtained, if there are no fiber-fiber interactions and the initial fiber orientation distribution is isotropic. Although all test cases considered in this paper fulfil the mentioned two criteria, we used the numerical IBOF closure to solve Jeffery's equation.

Due to the assumed incompressibility, only isochoric deformations are allowed for the introduced model. That means that $\overline{\boldsymbol{D}}$ is identical with its deviatoric part $\overline{\boldsymbol{D}}^{\prime}$. Jeffery's Eq. (7) and its extension to second order tensors (8) are valid for compressional cases, as well. Therefore, (7) and (8) are denoted here in the general form with $\bar{D}$.

\section{Deformation, anisotropy, dimensionless and reduced measures}

As a scalar deformation measure, the effective von Mises equivalent strain $\bar{\varepsilon}_{\mathrm{M}}$ is chosen which is defined by

$$
\bar{\varepsilon}_{\mathrm{M}}(t)=\int_{0}^{t} \dot{\bar{\varepsilon}}_{\mathrm{M}} \mathrm{dt} \quad, \quad \dot{\bar{\varepsilon}}_{\mathrm{M}}=\sqrt{\frac{3}{2}}\left\|\overline{\boldsymbol{D}}^{\prime}\right\| .
$$

The anisotropy measures $\left\|\boldsymbol{T}^{\text {aniso }}\right\|$ and $\left\|\mathbb{T}^{\text {aniso }}\right\|$ of an arbitrary second order tensor $\boldsymbol{T}$ and fourth order tensor $\mathbb{T}$, respectively, are calculated as

$$
\left\|\boldsymbol{T}^{\text {aniso }}\right\|=\left\|\boldsymbol{T}-\boldsymbol{T}^{\text {iso }}\right\|, \quad\left\|\mathbb{T}^{\text {aniso }}\right\|=\left\|\mathbb{T}-\mathbb{T}^{\text {iso }}\right\|,
$$

where

$$
\boldsymbol{T}^{\text {iso }}=\frac{1}{3}(\boldsymbol{T} \cdot \boldsymbol{I}) \boldsymbol{I} \quad, \quad \mathbb{T}^{\text {iso }}=\left(\mathbb{T} \cdot \mathbb{P}_{1}\right) \mathbb{P}_{1}+\frac{1}{5}\left(\mathbb{T} \cdot \mathbb{P}_{2}\right) \mathbb{P}_{2}
$$

are the isotropic parts of the corresponding tensors. It is assumed that $\mathbb{T}$ has the major and the minor symmetries. The scalar products $\boldsymbol{T} \cdot \boldsymbol{I}$ and $\mathbb{T} \cdot \mathbb{P}_{2}$ are calculated in Caresian coordinate systems by $T_{i j} I_{i j}$ and $T_{i j k l} P_{2 i j k l}$, respectively, with the use of Einstein's summation convention. Since the three-dimensional space is considered here, the summation indices are running from 1 to 3 . $\|$. denotes the Frobenius norm.

The following dimensionless $\left(\bar{\eta}_{\mathrm{s}}^{*}, \overline{\mathbb{V}}^{*}\right)$ and reduced $\left(\overline{\boldsymbol{\sigma}}^{*}\right)$ measures are used:

$$
\bar{\eta}_{\mathrm{s}}^{*}=\bar{\eta}_{\mathrm{s}} / \eta_{\mathrm{s}} \quad, \quad \overline{\mathbb{V}}^{*}=\overline{\mathbb{V}} / \eta_{\mathrm{s}} \quad, \quad \overline{\boldsymbol{\sigma}}^{*}=\overline{\boldsymbol{\sigma}} / \eta_{\mathrm{s}}
$$

where $\bar{\eta}_{\mathrm{S}}$ is the effective scalar shear viscosity of the mixture, determined through

$$
2 \bar{\eta}_{\mathrm{s}}=\frac{1}{5} \overline{\mathbb{V}} \cdot \mathbb{P}_{2} .
$$

\section{Phenomenological model for comparison}

In order to verify the proposed anisotropic material model (2), it is compared to the Dinh and Armstrong (1984) constitutive law (see also Latz et al. (2010)) through the numerically determined stress values. The reduced stress tensor $\overline{\boldsymbol{\sigma}}^{*}$ obtained by the introduced - mean field homogenization based - rheology model (2) and (13) is denoted for the comparison with $\bar{\sigma}_{\mathrm{mf}}$, and the equation through which it is defined, is given here for convenience

$$
\overline{\boldsymbol{\sigma}}_{\mathrm{mf}}^{*}=\overline{\mathbb{V}}\left[\overline{\boldsymbol{D}}^{\prime}\right] / \eta_{\mathrm{s}}
$$

To calculate the fully coupled stress in the flow of short fiber reinforced composites, Latz et al. (2010) uses the half phenomenological Dinh-Armstrong model which is given here for the case of incompressibility

$$
\overline{\boldsymbol{\sigma}}_{\mathrm{ph}}^{*}=\left(2 \bar{\eta}_{\mathrm{s}}\left(\mathbb{I}^{\mathrm{S}}+N_{\mathrm{p}} \overline{\mathbb{N}}\right)\left[\overline{\boldsymbol{D}}^{\prime}\right] / \eta_{\mathrm{s}}\right)^{\prime},
$$

where $N_{\mathrm{p}}$ is the coupling parameter and $\bar{\eta}_{\mathrm{s}}$ is the effective scalar shear viscosity of the mixture, determined for the comparison through (14). Please note, the difference lies in the fact that (16) is linear in $\overline{\mathbb{N}}$, but (15) is a function of $\bar{N}$ and $\overline{\mathbb{N}}$ through (4) as it is described in (Advani and Tucker III 1987).

Based on the model (15), an optimal $N_{\mathrm{p}}$ is determined for (16) with the least square approximation

$$
\frac{\mathrm{d}\left\|\overline{\boldsymbol{\sigma}}_{\mathrm{mf}}^{*}-\overline{\boldsymbol{\sigma}}_{\mathrm{ph}}^{*}\right\|}{\mathrm{d} N_{\mathrm{p}}}=0 \Rightarrow N_{\mathrm{p}}^{\mathrm{opt}}
$$

for every time step. $\bar{\sigma}_{\mathrm{ph}}^{*}$ calculated with the optimal coupling parameter $N_{\mathrm{p}}^{\mathrm{opt}}$ is compared to $\overline{\boldsymbol{\sigma}}_{\mathrm{mf}}^{*}$ in the Results section.

\section{Numerical implementation}

Only one point of the macro-continuum is investigated with stationary velocity gradient. $M$ pieces of randomly generated, equally distributed discrete orientation vectors (Mardia and Jupp 2000), immersed in the liquid matrix, are investigated.

The differential equations of the fiber reorientation (7) and (9) and the deformation measure (10) are discretised and numerically integrated with the use of the explicit one-step method. The new orientation vectors are in each time step normalized

$$
\boldsymbol{n}_{\alpha}(t+\Delta t)=\frac{\boldsymbol{n}_{\alpha}(t)+\dot{\boldsymbol{n}}_{\alpha}(t) \Delta t}{\left\|\boldsymbol{n}_{\alpha}(t)+\dot{\boldsymbol{n}}_{\alpha}(t) \Delta t\right\|},
$$

and it is ensured that the new second order fiber orientation tensor has unit trace

$$
\overline{\mathbf{N}}(t+\Delta t)=\frac{\overline{\mathbf{N}}(t)+\dot{\overline{\mathbf{N}}}(t) \Delta t}{\operatorname{tr}(\overline{\mathbf{N}}(t)+\dot{\overline{\mathbf{N}}}(t) \Delta t)} .
$$


The model calculated based on (18), (7) and (8) is called a discrete model. To calculate (19) with the use of (9) (and with isotropic initial fiber orientation tensor $\overline{\boldsymbol{N}}(0)$ ), a closure approximation is needed to approximate $\overline{\mathbb{N}}$ based on $\bar{N}$ in each time step, since otherwise the expression (9) is undetermined. The models based on (19) calculated with quadratic closure and with IBOF closure are called here quadratic model and IBOF model, respectively.

The von Mises equivalent strain, determined through

$$
\bar{\varepsilon}_{\mathrm{M}}(t+\Delta t)=\bar{\varepsilon}_{\mathrm{M}}(t)+\dot{\bar{\varepsilon}}_{\mathrm{M}}(t) \Delta t
$$

is exact, because the velocity gradient is considered to be constant. The time step size $\Delta t$ was decreased until the computations with the time increments $2 \Delta t, \Delta t$ and $\Delta t / 2$ gave the same results.

The chosen parameters for the simulation are listed in Table 1. The discrete reference model is evaluated with $M=1000$ fibers. For short fibers, the infinite aspect ratio is not realistic. In spite of that, the numerically more challenging limit case (infinite aspect ratio) is investigated, assuming immensely slender fibers. This induces, that the geometry parameter $\bar{\xi}=1$ in the Eqs. (7) and (9). $\bar{a}$ influences the polarization tensor $\mathbb{P}_{\alpha}$, as well. Because of the limit cases of immensely slender fibers $(\bar{a} \rightarrow \infty)$ and incompressibility $\left(\eta_{\mathrm{v}} \rightarrow \infty\right), \mathbb{P}_{\alpha}$ has two zero eigenvalues and it is inverted on its non-singular subspace for (5), to avoid the blow up of $\overline{\mathbb{V}}^{\text {ud }}$. The matrix shear viscosity $\eta_{\mathrm{s}}$ is chosen to be $1 \mathrm{~Pa}$ s, because the homogenization equation for $\overline{\mathbb{V}}$ is linear in $\bar{\eta}_{\mathrm{s}}$. This means that $\overline{\mathbb{V}}\left(\bar{\eta}_{\mathrm{s}}\right)=$ $\bar{\eta}_{\mathrm{s}} \overline{\mathbb{V}}(1)$ and $\overline{\mathbb{V}}^{*}=\overline{\mathbb{V}}(1)$. The calculations are carried out with $c_{\mathrm{f}}=30 \%$ fiber volume fraction which is realistic for sheet mould compounds with resin matrix, but not for composites with a thermoplastic matrix.

\section{Investigated, representative flow modes}

To validate the above described model, four representative isochoric flow modes are investigated: shear flow (sf), elongational flow (ef), compressional flow (cf), and a planar flow (pf) which is the superposition of an elongational and a compressional flow. The corresponding velocity gradients are given by

Table 1 Material parameters for the simulations

\begin{tabular}{lll}
\hline Symbol & Value & Unit \\
\hline$M$ & 1000 & - \\
$\bar{a}$ & $\infty$ & - \\
$\eta_{\mathrm{v}}$ & $\infty$ & Pas \\
$\eta_{\mathrm{s}}$ & 1 & Pas \\
$c_{\mathrm{f}}$ & 0.3 & - \\
\hline
\end{tabular}

$$
\begin{aligned}
& \overline{\boldsymbol{L}}^{\mathrm{sf}} \hat{=}\left(\begin{array}{ccc}
0 & b & 0 \\
0 & 0 & 0 \\
0 & 0 & 0
\end{array}\right), \quad \overline{\boldsymbol{L}}^{\mathrm{ef}} \hat{=}\left(\begin{array}{ccc}
b & 0 & 0 \\
0 & -\frac{b}{2} & 0 \\
0 & 0 & -\frac{b}{2}
\end{array}\right), \\
& \overline{\boldsymbol{L}}^{\mathrm{cf}} \hat{=}\left(\begin{array}{ccc}
-b & 0 & 0 \\
0 & \frac{b}{2} & 0 \\
0 & 0 & \frac{b}{2}
\end{array}\right), \overline{\boldsymbol{L}}^{\mathrm{pf}} \hat{=}\left(\begin{array}{ccc}
b & 0 & 0 \\
0 & -b & 0 \\
0 & 0 & 0
\end{array}\right),
\end{aligned}
$$

where $b$ is an arbitrary scalar factor which determines also the magnitude of the strain rate. $b$ has the dimension $\mathrm{s}^{-1}$. The simulations are carried out with $b=1 \mathrm{~s}^{-1}$.

\section{Results}

\section{Discrete pole figures}

For the four representative flow modes, firstly the fiber reorientations are investigated on discrete pole figures, see Fig. 1. Each row belongs to one of the investigated flow types. In the initial state - the most left pole figures in Fig. $1, \bar{\varepsilon}_{\mathrm{M}}=0$ - the 1000 directions have a randomly generated uniform distribution (Mardia and Jupp 2000). The second and third pole figure in a row in Fig. 1 represent the fiber orientation distribution at $\bar{\varepsilon}_{\mathrm{M}}=3$ and $\bar{\varepsilon}_{\mathrm{M}}=6$, respectively. In the fourth pole figure in a row, the quasistationary orientation distributions are plotted at $\bar{\varepsilon}_{\mathrm{M}}=9$. These pole figures indicate that the quasi-stationary orientation state for the shear flow and for the compressional flow is nearly planar and planar, respectively. The quasistationary orientation state for the elongational flow and for the planar flow is unidirectional and nearly unidirectional, respectively. A periodic solution does not appear here, because the aspect ratio of the representative fiber is set to infinity. For this case, the stationary solution is also stated, e.g., by (Jeffery 1922) and (Junk and Illner 2007). The approximation of the fiber reorientations with fiber orientation tensors is discussed in the next subsection.

\section{Component plots}

In the following, the component plots of the second$(\overline{\boldsymbol{N}})$ and fourth- $(\overline{\mathbb{N}})$ order fiber orientation tensor, of the dimensionless effective viscosity tensor $\left(\overline{\mathbb{V}}^{*}\right)$ and of the reduced effective stress tensor $\left(\overline{\boldsymbol{\sigma}}^{*}\right)$ are shown and described, for the four investigated flow cases.

\section{Shear flow}

In Fig. 2, the component plots for the shear flow are depicted. The initial fiber orientation state is isotropic, this is represented also in the second- and fourth order fiber orientation tensor component plots in Fig. 2a and $b$. The transient change of the fiber orientation is anisotropic, and it is not transversely isotropic. In the case of the shear flow the stationary state is not reached until $\bar{\varepsilon}_{\mathrm{M}}=15$. The quasi-stationary orientation state is nearly planar. In Fig. 2c, the transient change of the components of the dimensionless fourth order effective viscosity tensor $\overline{\mathbb{V}}^{*}$ is plotted. It is also isotropic in the 

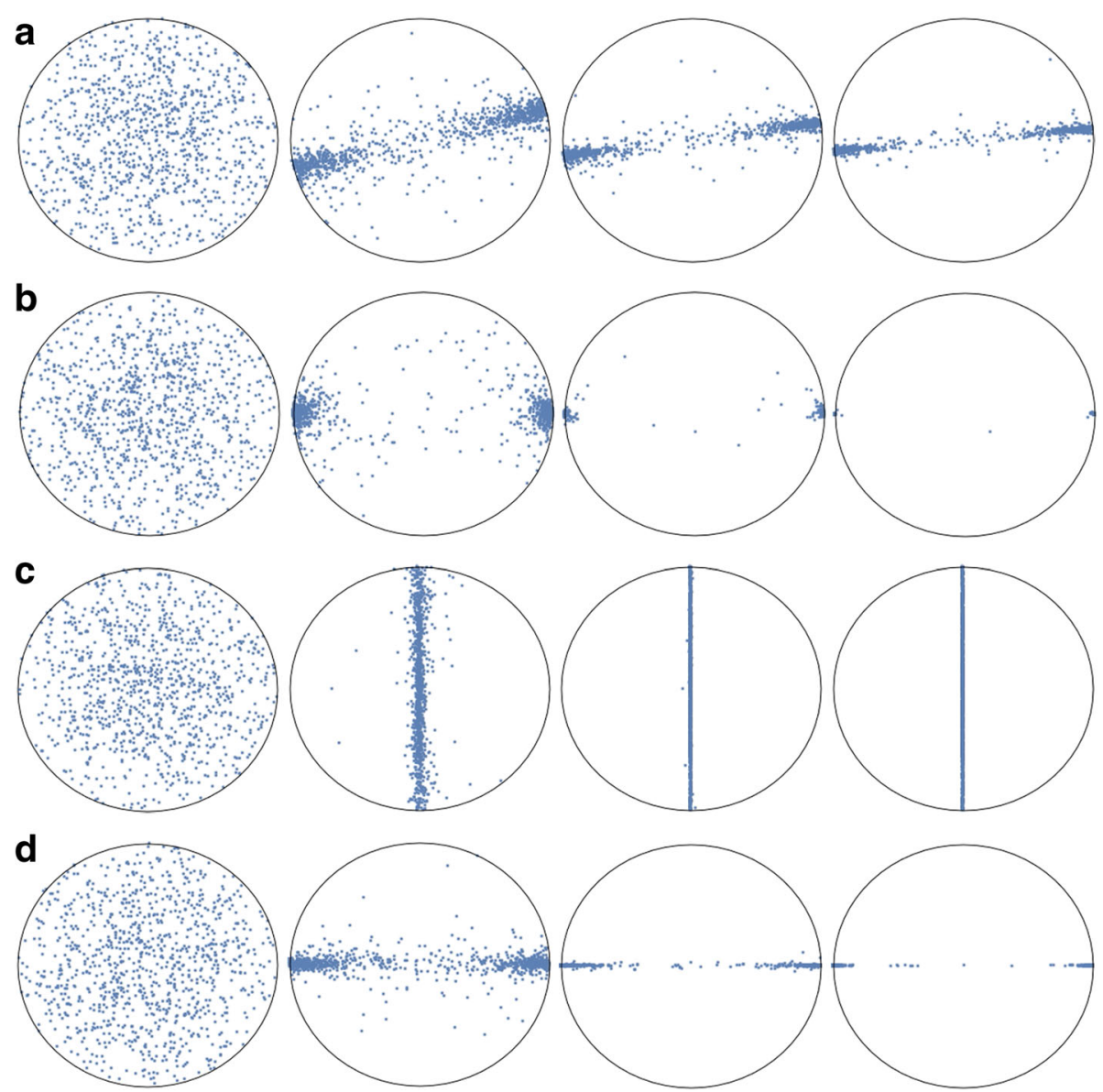

Fig. 1 Stereographic pole figures. Evolution of 1000 orientations on pole figures. The initial orientation state $\left(\bar{\varepsilon}_{\mathrm{M}}=0\right)$ is in all investigated cases a randomly generated uniform distribution (left). The other pole figures show the orientation state at $\bar{\varepsilon}_{\mathrm{M}}=3, \bar{\varepsilon}_{\mathrm{M}}=6$ and $\bar{\varepsilon}_{\mathrm{M}}=9$ (right) (a) Orientation evolution in shear flow (b) Orientation evolution in elongational flow (c) Orientation evolution in compressional flow (d) Orientation evolution in planar flow

beginning and evolves anisotropy due to the flow. The Cauchy stress tensor is anisotropic during the complete investigated deformation process, as it is shown in Fig. 2d.

The component plot curves of $\overline{\mathbb{N}}$ graphically do not represent more information for the reader than the component plot curves of $\bar{N}$, although $\overline{\mathbb{N}}$ contains more information with respect to the anisotropy of the orientation distribution than $\bar{N}$. For this reason, for the other three investigated flow cases, only the component plots of $\overline{\boldsymbol{N}}, \overline{\mathbb{V}}^{*}$ and $\overline{\boldsymbol{\sigma}}^{*}$ are given in the following.

\section{Comparison of the flow cases}

First, the components of $\bar{N}$ and $\overline{\mathbb{V}}^{*}$ are presented for all four investigated flow cases in Fig. 3, where the corresponding component plots of the shear flow case are repeated in order to help comparison.

The reorientation of the fibers in the investigated elongational flow is faster than in the shear flow.
The stationary state is reached in this case already at about $\bar{\varepsilon}_{\mathrm{M}}=6$, as Fig. 3c and $\mathrm{d}$ show. The transient change is transversely isotropic, and the stationary case is unidirectional considering the fiber orientation.

In the case of the compressional flow, the reorientation of the fibers is even faster than in the flow cases mentioned before. The stationary state is already reached at about $\bar{\varepsilon}_{\mathrm{M}}=4$, as it is shown in Fig. 3e and f. The transient change is, here, also transversely isotropic, as in the elongational flow, but the end state is, here, a planar isotropic one.

The investigated planar flow is a combination of an elongational and a compressional flow. As Fig. $3 g$ and $h$ show, the reorientation of the fibers is slower than in the two investigated cases before, the stationary state is reached only at about $\bar{\varepsilon}_{\mathrm{M}}=8$. The transient change is, here, not transversely isotropic similarly as in the shear flow. In the stationary case, the fiber orientation is unidirectional. 

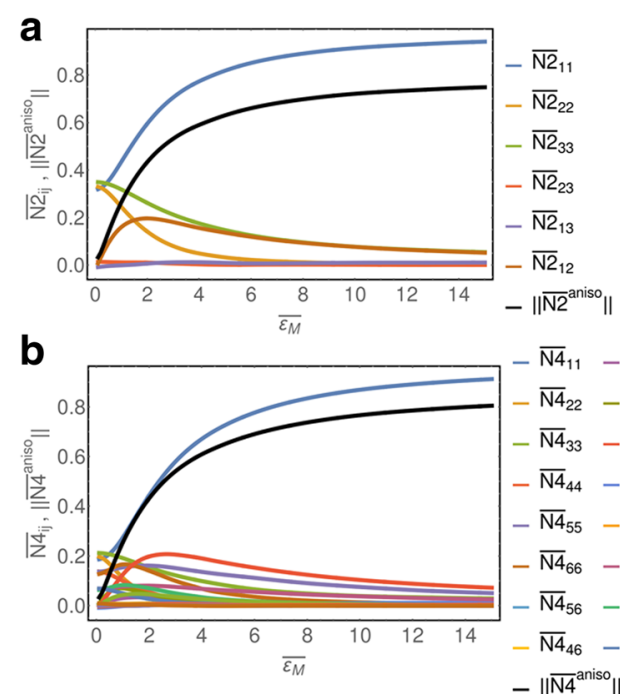

$-\overline{\mathrm{N}}_{11}-\overline{\mathrm{N}}_{36}-\overline{\mathrm{N}}_{34}$
$-\overline{\mathrm{N}}_{22}-\overline{\mathrm{N}}_{26}-\overline{\mathrm{N}}_{45}$
$-\overline{\mathrm{N}}_{33}-\overline{\mathrm{N}}_{16}-\overline{\mathrm{N}}_{35}$
$-\overline{\mathrm{N}}_{44}-\overline{\mathrm{N}}_{15}-\overline{\mathrm{N}}_{25}$
$-\overline{\mathrm{N}}_{55}-\overline{\mathrm{N}}_{14}-\overline{\mathrm{N}}_{24}$
$-\overline{\mathrm{N}}_{66}-\overline{\mathrm{N4}}_{13}$
$-\overline{\mathrm{N}}_{56}-\overline{\mathrm{N}}_{12}$
$-\overline{\mathrm{N}}_{46}-\overline{\mathrm{N}}_{23}$
$-\| \overline{\mathrm{N4}}^{\text {aniso }}$

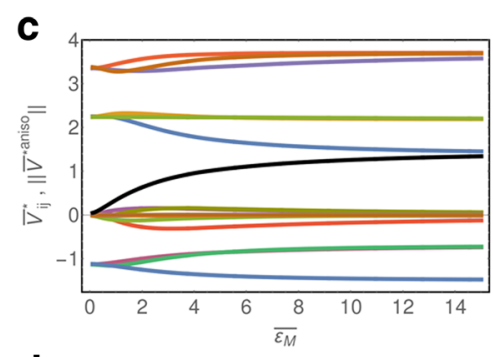

$-\bar{V}_{11}^{*}-\bar{V}_{36}^{*}-\bar{V}_{34}^{*}$

d

$-\bar{V}_{22}^{*}-\bar{\nabla}_{26}^{*}-\bar{\nabla}_{45}^{*}$

$-\bar{V}_{33}^{*}-\bar{V}_{16}^{*}-\bar{V}_{35}^{*}$

$-\bar{V}_{44}^{*}-\bar{V}_{15}^{*}-\bar{V}_{25}^{*}$

$-\bar{V}_{55}^{*}-\bar{V}_{14}^{*}-\bar{V}_{24}^{*}$

$-\bar{V}_{66}^{*}-\bar{V}_{13}^{*}$

$-\bar{V}_{56}^{*}-\bar{V}_{12}^{*}$

$\bar{V}_{46}^{*}-\bar{V}_{23}^{*}$

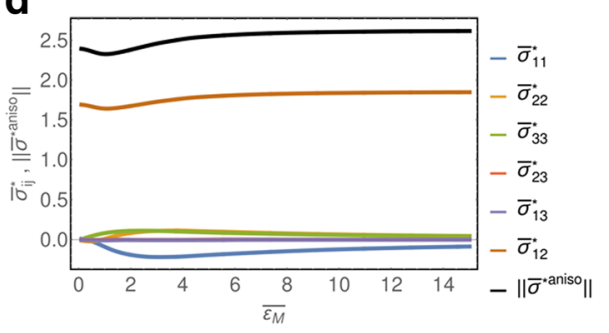

Fig. 2 Component plots - Shear flow. The component plots of the second- $(\overline{\boldsymbol{N}})$ and fourth- $(\overline{\mathbb{N}})$ order orientation tensors, and of the dimensionless effective viscosity tensor $\left(\overline{\mathbb{V}}^{*}\right)$ and of the reduced stress tensor $\left(\overline{\boldsymbol{\sigma}}^{*}\right)$ vs. the von Mises equivalent strain rate $\left(\bar{\varepsilon}_{\mathrm{M}}\right)$, for the case of a shear flow (a) Components of $\overline{\boldsymbol{N}}$, shear flow (b) Components of $\overline{\mathbb{N}}$, shear flow (c) Components of $\overline{\mathbb{V}}^{*}$, shear flow (d) Components of $\overline{\boldsymbol{\sigma}}^{*}$, shear flow

\section{Comparison of the two models}

The differences and similarities between the models (15) and (16) with $N_{\mathrm{p}}^{\mathrm{opt}}$ for $c_{\mathrm{f}}=0.3$ are shown in Fig. $4 \mathrm{a}-\mathrm{d}$. The continuous lines represent the mean field theory based model (the discrete model and the IBOF model results in the same curves, as discussed in the next section), and the dashed lines the phenomenological one. The biggest difference is observed in the case of the simple shear flow, see Fig. 4a. In the case of the elongational and the compressional flow, the two models (15) and (16) behave identically, in Fig. 4b and c, the dashed lines cover the continuous ones. Regarding the investigated planar flow, the difference between the two models is smaller than in the case of the simple shear flow. Based on the results shown in Fig. 4, the similar behaviour of the mean field theory based model (15) and the phenomenological model (16) is stated. This statement is obtained assuming an incompressible material behaviour.

\section{Discussion}

Relative calculation times and closure approximation Implementing the orientation evolution with discrete fibers (7) or with the help of fiber orientation tensors (9) notably influences the calculation time. Figure $5 \mathrm{a}$ shows the difference of the calculation times of a discrete model with 1000 fibers, and the model with fiber orientation tensors with the quadratic closure (Doi 1981) 


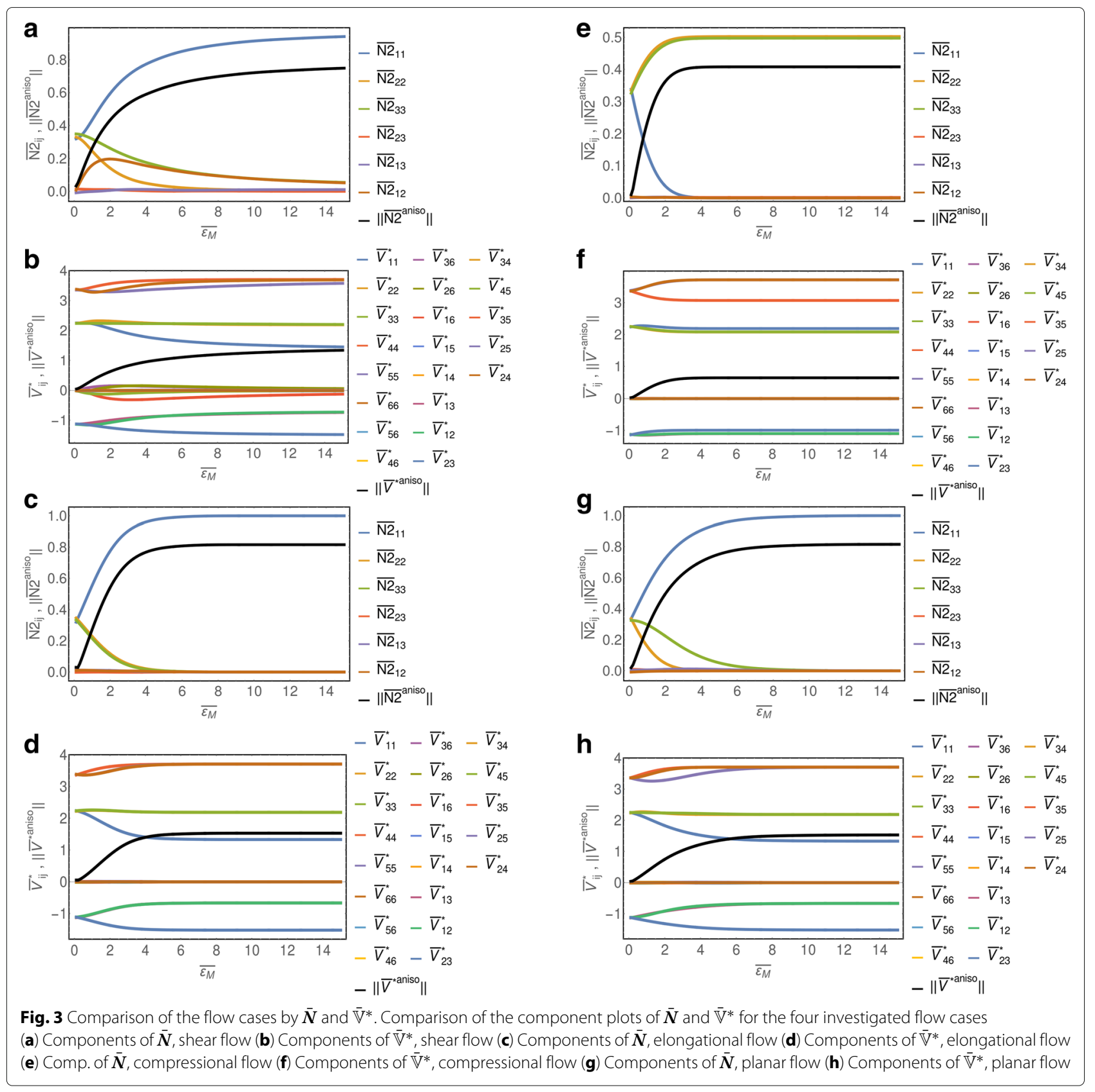

relative to the model with the IBOF closure (Chung et al. 2002). The model with the 1000 discrete fibers is about 8 times slower, and the model with the quadratic closure about 3 times faster, than the model with the IBOF closure. Although the model with the quadratic closure is the fastest one out of the three investigated models, the use of it is not recommended, because it provides qualitatively different results during the transient change, than the discrete model which is treated as reference (see Fig. 5b and c). The quadratic closure is exact for highly aligned fibers, but not for arbitrary fiber orientation distributions. The calculation with the IBOF closure is slower than that with quadratic closure, but it provides qualitatively the same results also during the transient change as the discrete reference model (see Fig. $5 b$ and d). The differences and similarities are plotted in Fig. 5 only for the case of a shear flow (21). Similar qualitative differences and similarities are observed also for the three other investigated flow cases. Because of the above mentioned reasons, the use of the fiber orientation tensors with the IBOF closure is recommended for use. 

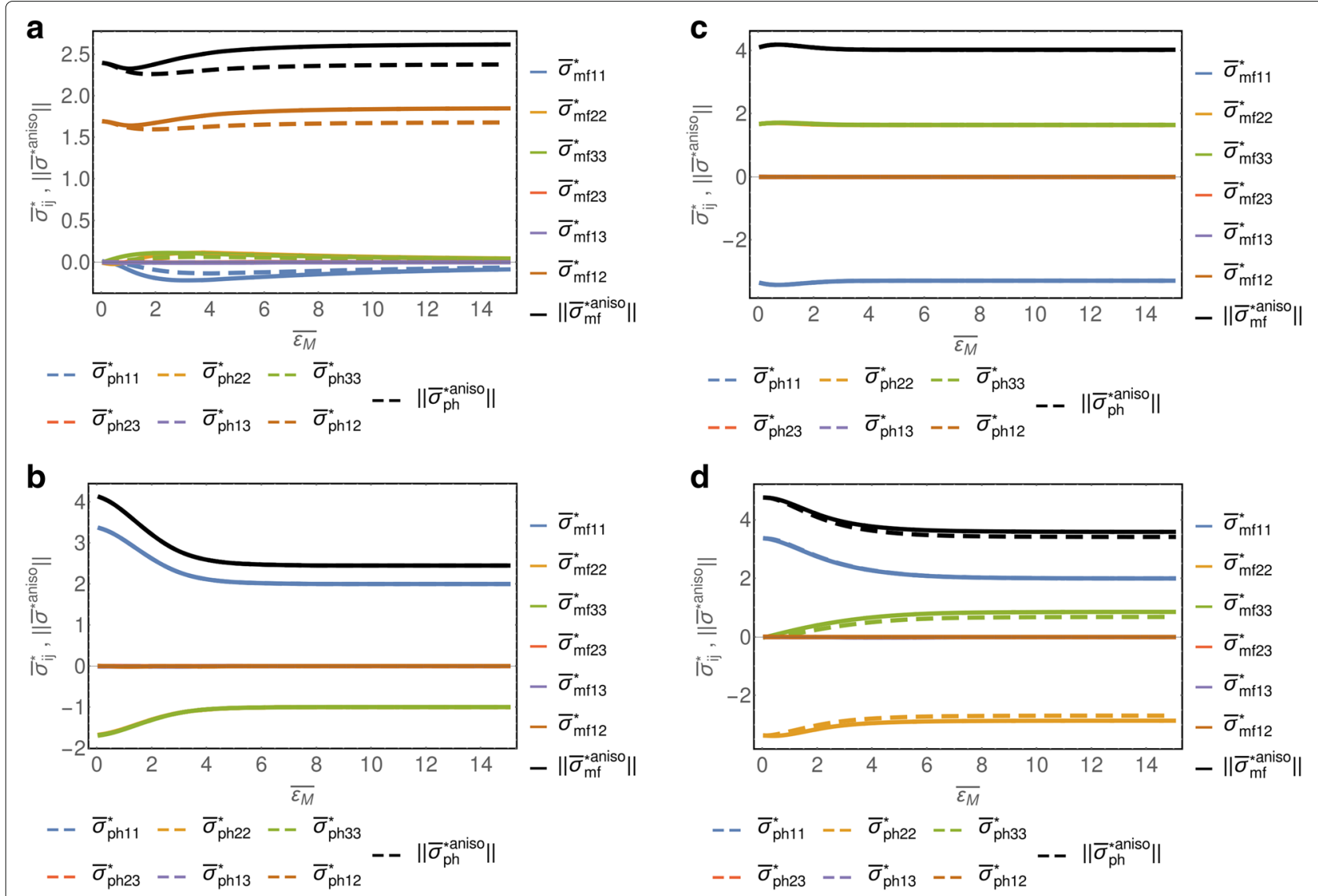

Fig. 4 Comparison of the two models. The components of the stress tensors are compared in these figures for the four investigated flow cases. The continuous lines represent the homogenized model and the dashed lines the model with the optimal coupling parameter $N_{\mathrm{p}}^{\text {opt }}$ (a) Shear flow (b) Elongational flow (c) Compressional flow (d) Planar flow

\section{Sensitivity of the viscosity}

Instead of discussing the component plots of the four investigated cases in detail, a coherent, artificial parametrization of $\overline{\boldsymbol{N}}, \overline{\mathbb{N}}$ and $\overline{\mathbb{V}}^{*}$ is considered, with the use of the parameter $\lambda$. The parametrization

$$
\bar{N}(\lambda)=\left(\begin{array}{ccc}
\frac{1}{3}+\lambda & 0 & 0 \\
& \frac{1}{3}-\frac{\lambda}{2} & 0 \\
\text { sym. } & & \frac{1}{3}-\frac{\lambda}{2}
\end{array}\right)
$$

is set up such that $\lambda=0$ describes the isotropic fiber orientation and $\lambda=2 / 3$ the unidirectional one. $\overline{\mathbb{N}}(\lambda)$ is calculated here with the IBOF closure, and $\overline{\mathbb{V}}^{*}(\lambda)$ with the two-step homogenization described in this paper, based on the fiber orientation tensors $\bar{N}(\lambda)$ and $\overline{\mathbb{N}}(\lambda)$. Notice that $\bar{N}(\lambda)$ is linear, but $\overline{\mathbb{N}}(\lambda)$ and $\overline{\mathbb{V}}^{*}(\lambda)$ are not linear in $\lambda$, see Fig. 6a-c. It is difficult to interpret the change of the components of $\overline{\mathbb{V}}^{*}$ also in Fig. 3b, d, f, h and in Fig. 6c. To demonstrate the meaning of the change of the components of $\overline{\mathbb{V}}^{*}$, dimensionless effective scalar viscosities are extracted from the dimensionless effective viscosity tensor, analogously as it is done in (T Böhlke 2001) for the shear modulus. For this extraction, the definition of a plane and of a direction in this plain is necessary. Let $\boldsymbol{p}$ denote the normal vector of the plane, and $\boldsymbol{d}$ denote a chosen direction in this plane. The dimensionless effective shear viscosity in the plane $\boldsymbol{p}$, in the direction $\boldsymbol{d}$ is $\bar{\eta}_{\mathrm{s}}^{*}(\boldsymbol{p}, \boldsymbol{d})$ which is determined by

$$
\begin{aligned}
\bar{\eta}_{\mathrm{s}}^{*}(\boldsymbol{p}, \boldsymbol{d})= & \frac{1}{2 \boldsymbol{M}(\boldsymbol{p}, \boldsymbol{d}) \cdot\left(\overline{\mathbb{V}}^{*}\right)^{-1}[\boldsymbol{M}(\boldsymbol{p}, \boldsymbol{d})]}, \\
& \boldsymbol{M}(\boldsymbol{p}, \boldsymbol{d})=\sqrt{2}(\boldsymbol{p} \otimes \boldsymbol{d}+\boldsymbol{d} \otimes \boldsymbol{p}) / 2 .
\end{aligned}
$$

Note that $\overline{\mathbb{V}}^{*}$ is singular, because of the assumed incompressibility and it is inverted on its non-singular subspace, analogously as $\mathbb{P}_{\alpha}$ is inverted for (5). The dimensionless effective scalar viscosity $\bar{\eta}_{\mathrm{s}}^{*}(\boldsymbol{p}, \boldsymbol{d})$ can be imagined as a four-dimensional surface. The three independent coordinates of $\bar{\eta}_{\mathrm{s}}^{*}(\boldsymbol{p}, \boldsymbol{d})$ are the two angles determining $\boldsymbol{p}$ and one angle determining $\boldsymbol{d}$ in the plane. The fourth, dependent variable is $\bar{\eta}_{\mathrm{s}}^{*}$. Instead of the four-dimensional surface, a two-dimensional representative slice of it is depicted in Fig. 6d which represents $\bar{\eta}_{\mathrm{s}}^{*}(\boldsymbol{p}, \boldsymbol{d})$ for the case that both $\boldsymbol{p}$ and $\boldsymbol{d}$ lie in the $x-y$ plane. In Fig. $6 \mathrm{~d}, \boldsymbol{p}$ is the radial direction, and $\boldsymbol{d}$ is tangential to it $(\boldsymbol{p} \cdot \boldsymbol{d}=0)$. 

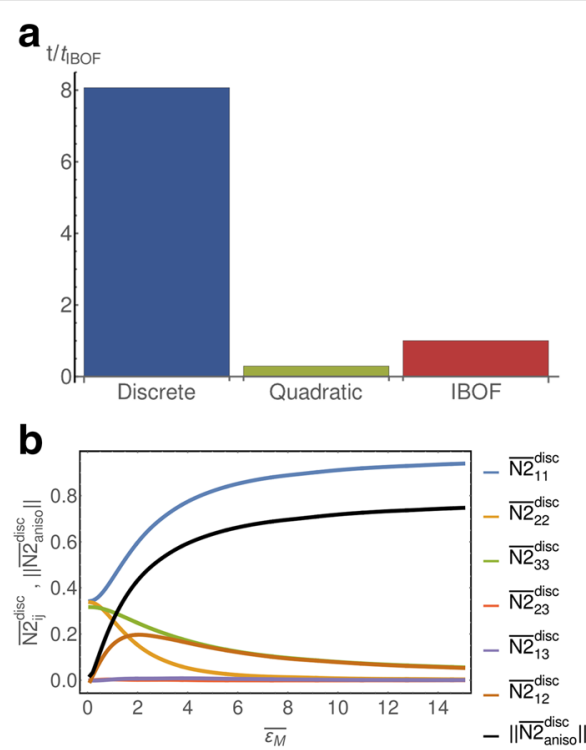

C

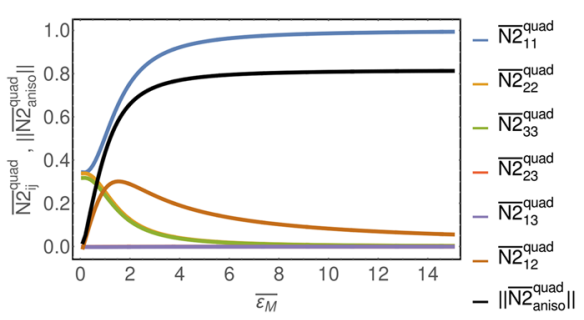

d

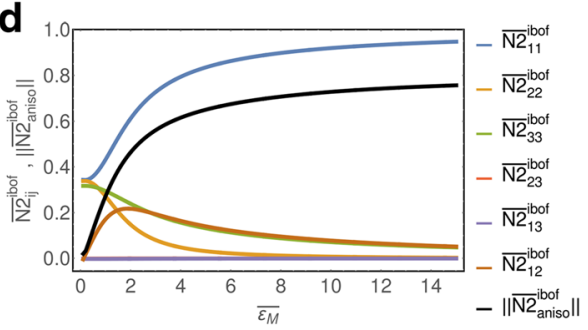

Fig. 5 Relative calculation times and closure approximation. The calculation time of the discrete model and of the model with quadratic closure relative to the calculation time of the model with IBOF closure is depicted in subfigure $\mathbf{a}$. The subfigures $\mathbf{b}, \mathbf{c}$ and $\mathbf{d}$ represent the evolution of the components of the second order fiber orientation tensor $\overline{\boldsymbol{N}}$ for the case of a shear flow, with identical isotropic initial conditions. Notice the qualitative difference and similarity between $\mathbf{b}-\mathbf{c}$ and $\mathbf{b}-\mathbf{d}$, respectively (a) Relative calculation times (b) Evolution of $\overline{\boldsymbol{N}}$ with discrete model (c) Evolution of $\overline{\boldsymbol{N}}$ with quadratic closure (d) Evolution of $\overline{\boldsymbol{N}}$ with IBOF closure
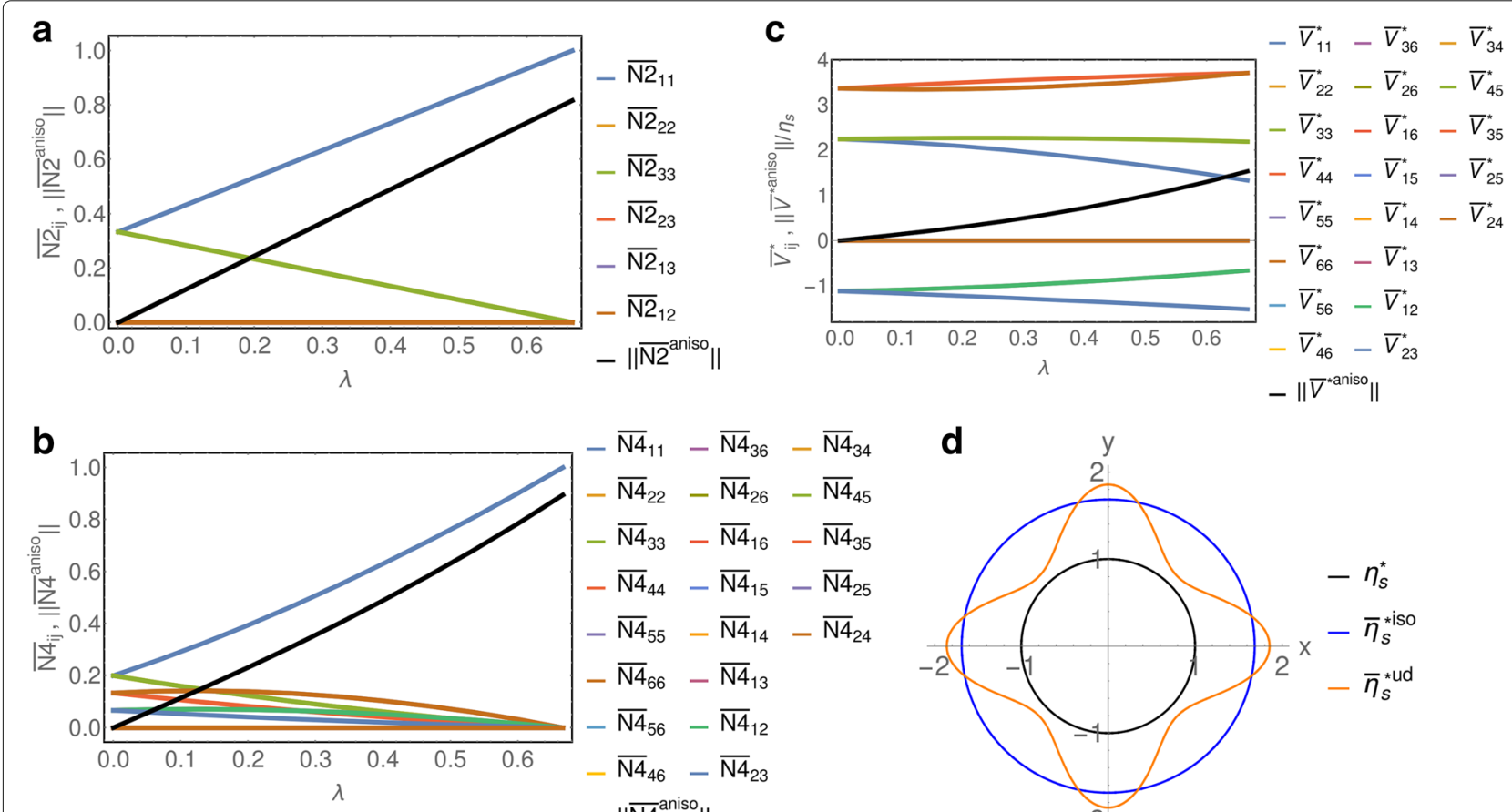

$$
\begin{aligned}
& -\overline{\mathrm{N}}_{11}-\overline{\mathrm{N}}_{36}-\overline{\mathrm{N}}_{34} \\
& -\overline{\mathrm{N}}_{22}-\overline{\mathrm{N}}_{26}-\overline{\mathrm{N}}_{45} \\
& -\overline{\mathrm{N}}_{33}-\overline{\mathrm{N}}_{16}-\overline{\mathrm{N}}_{35} \\
& -\overline{\mathrm{N}}_{44}-\overline{\mathrm{N}}_{15}-\overline{\mathrm{N}}_{25} \\
& -\overline{\mathrm{N}}_{55}-\overline{\mathrm{N}}_{14}-\overline{\mathrm{N}}_{24} \\
& -\overline{\mathrm{N}}_{66}-\overline{\mathrm{N4}}_{13} \\
& -\overline{\mathrm{N}}_{56}-\overline{\mathrm{N}}_{12} \\
& -\overline{\mathrm{N}}_{46}-\overline{\mathrm{N}}_{23} \\
& -\left\|\overline{\mathrm{N}}^{\text {aniso }}\right\|
\end{aligned}
$$

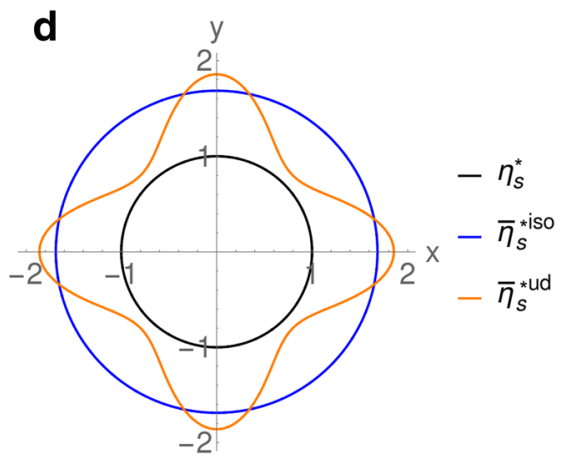

Fig. 6 Sensitivity of the viscosity. The component of $\overline{\boldsymbol{N}}, \overline{\mathbb{N}}$ and $\overline{\mathbb{V}}^{*}$ in dependence of the parameter $\lambda$. In the case of $\lambda=0$ the fiber orientation distribution is isotropic, and by $\lambda=2 / 3$ it is unidirectional. Subfigure (d) show the direction dependent shear viscosity in the $x-y$ plane, for the isotropic, and the unidirectional case $(\mathbf{a})$ Components of $\overline{\boldsymbol{N}}(\lambda)(\mathbf{b})$ Components of $\overline{\mathbb{N}}(\lambda)(\mathbf{c})$ Components of $\overline{\mathbb{V}}^{*}(\lambda)(\mathbf{d}) \bar{\eta}_{\mathrm{s}}^{*}(\boldsymbol{p}, \boldsymbol{d})$ for iso. and ud. case 
The three curves represent the matrix (black circle), the effective isotropic $\left(c_{\mathrm{f}}=30 \%\right)$ and the effective unidirectional (in $x$-direction) scalar viscosity belonging to the proper $\boldsymbol{p}$-s and $\boldsymbol{d}$-s. The introduced model predicts that due to adding $30 \mathrm{~V} / \mathrm{V} \%$ fibers to the matrix material, the effective isotropic viscosity increases to 1.7 -fold of the matrix viscosity (blue circle). Considering the unidirectional fiber orientation state in $x$-direction, two maximum and two minimum "directions" are observed (orange curve). The maximum is about 1.9 -fold matrix viscosity and the minimum is about 1.1 -fold matrix viscosity. The model predicts the two maximum "directions" in fiber direction and perpendicular to it. The minimum "directions" have the angle $\pm 45^{\circ}$ to the fiber direction.

Beside the actual fiber orientation distribution and the velocity field of the flow, the local fiber volume content, the spatial and temporal change of the temperature, together with the curing grade, have also remarkable effects on the effective viscosity. These effects can be taken into account with the extension of the introduced model by making $V^{\mathrm{m}}$ and $\overline{\boldsymbol{V}}^{\mathrm{ud}}$ dependent on the listed effects.

\section{Conclusions}

A mean field theory based effective linear viscosity model is introduced for short fiber reinforced polymers based on a two-step homogenization method. The presented model can handle arbitrary anisotropy of the effective viscosity which is caused by the evolving orientation distribution of the fibers. The numerical implementation of the introduced model with fiber orientation tensors calculates about eight times faster than the same model calculating with 1000 fiber orientation vectors. The use of the fiber orientation tensors is highly recommended for an efficient calculation even with the arising closure approximation. The effective direction dependent scalar shear viscosity is 1.1 to 1.8 times higher than the matrix shear viscosity at $30 \%$ fiber volume fraction. For each matrix-fiber suspension, the effect of the direction dependency has to be compared to the effect of the temperature change and to the effect of the deformation rate dependency of the matrix shear viscosity to decide whether the direction dependency has a remarkable effect on the flow of the suspension, or not. With the assumptions that the matrix material is incompressible and the fibers are rigid, the presented model gives qualitatively the same results as the phenomenological model used for comparison. A possible way to consider the anisotropic viscosity of fiber reinforced polymers is given in this paper.

\section{Abbreviations}

FODF: Fiber orientation distribution function; FRP: Fiber reinforced polymer; IBOF: Invariant based optimal fitting; TIF: Transversely isotropic fluid

\section{Acknowledgements}

This work was partially supported by the German Research Foundation (DFG) within the International Research Training Group "Integrated engineering of continuous-discontinuous long fiber reinforced polymer structures" (GRK 2078).

Funding

German Research Foundation, Grant Number: DFG GRK 2078/1.

\section{Authors' contributions}

RB carried out this study and drafted the manuscript. TB supervised the research, planned the study, contributed to the interpretation of results and writing of the manuscript. Both the authors read and approved the manuscript.

\section{Competing interests}

The authors declare that they have no competing interests.

Received: 12 October 2016 Accepted: 19 December 2016

Published online: 17 January 2017

\section{References}

Advani S, Tucker III C (1987) The use of tensors to describe and predict fiber orientation in short fiber composites. J Rheol 31(8):751-784

Castaneda P, Suquet P (1998) Nonlinear composites. Adv Appl Mech 34(998):171-302

Cowin S, Mehrabadi M (1987) On the identification of material symmetry for anisotropic elastic materials. Q J Mech Appl Math 40(4):451-476

Chung DH, Kwon TH, et al (2002) Invariant-based optimal fitting closure approximation for the numerical prediction of flow-induced fiber orientation. J Rheol 46(1):169-194

Dinh SH, Armstrong RC (1984) A rheological equation of state for semi-concentrated fiber suspensions. J Rheol 28:207-227

Doi M (1981) Molecular dynamics and rheological properties of concentrated solutions of rodlike polymers in isotropic and liquid crystalline phases. J Polym Sci Polym Phys Ed 19:243

Ericksen JL (1960) Anistropic fluids. Arch Rat Mech Anal 4:231-237

Ferry JD (1980) Viscoelastic Properties of Polymers. John Wiley \& Sons, New York

Folgar F, Tucker III C (1984) Orientation behavior of fibers in concentrated suspensions. J Reinf Plast Compos 3(2):98-119

Jeffery $G$ (1922) The motion of ellipsoidal particles immersed in a viscous fluid. Proc R Soc Lond Series A 102(715):161-179

Junk M, Illner R (2007) A new derivation of Jeffery's equation. J Math Fluid Mech 9(4):455-488

Kammoun S, Doghri I, Adam L, Robert G, Delannay L (2011) First pseudo-grain failure model for inelastic composites with misaligned short fibers. Compos A: Appl Sci Manuf 42(12):1892-1902

Kanatani KI (1984) Distribution of directional data and fabric tensors. Int J Eng Sci 22(2):149-164

Latz A, Strautins U, Niedziela D (2010) Comparative numerical study of two concentrated fiber suspension models. J Non-Newtonian Fluid Mech 165(13-14):764-781

Mardia KV, Jupp PE (2000) Directional Statistics. Wiley, Chichester [u.a.]

Montgomery-Smith S, Jack D, Smith DE (2011) The fast exact closure for jeffery's equation with diffusion. J Non-Newtonian Fluid Mech 166(7):343-353

Ospald F (2014) Numerical simulation of injection molding using OpenFOAM. Proc Appl Math Mech 14(1):673-674

Phan-Thien N, Graham AL (1991) A new constitutive model for fiber suspensions: flow past a sphere. Rheol Acta 30:44-57

Phelps J, Tucker III C (2009) An anisotropic rotary diffusion model for fiber orientation in short-and long-fiber thermoplastics. J Non-Newtonian Fluid 156(3):165-176

Poslinski A, Ryan M, Gupta R, Seshadri S, Frechette F (1988) Rheologica behavior of filled polymeric systems i. yield stress and shear-thinning effects. J Rheol (1978-present) 32(7):703-735

Schneider M, Ospald F, Kabel M (2015) Computational homogenization of elasticity on a staggered grid. Int J Numer Methods Eng 105(9):693-720

T Böhlke CB (2001) Graphical representation of the generalized Hooke's law. Tech Mech 21(2):145-158 
Walpole L (1969) On the overall elastic moduli of composite materials. J Mech Phys Solids 17(4):235-251

Wang J, O'Gara J, Tucker III C (2008) An objective model for slow orientation kinetics in concentrated fiber suspensions: Theory and rheological evidence. J Rheol 52(5):1179-1200

Willis JR (1977) Bounds and self-consistent estimates for the overall properties of anisotropic composites. J Mech Phys Solids 25:185-202

\section{Submit your manuscript to a SpringerOpen ${ }^{\circ}$} journal and benefit from:

- Convenient online submission

- Rigorous peer review

- Immediate publication on acceptance

- Open access: articles freely available online

- High visibility within the field

- Retaining the copyright to your article

Submit your next manuscript at $>$ springeropen.com 